\title{
Can subjects maintain a constant criterion in a memory task?
}

\author{
ALICE F. HEALY \\ Yale University, New Haven, Connecticut 06520 \\ and \\ CINDY JONES \\ Rockefeller University, New York, New York 10021
}

\begin{abstract}
In two recognition memory experiments, subjects rated how certain they were that a given test item had been shown previously. Subjects either received standard instructions about their rating criteria or were explicitly instructed to maintain criteria defined in the manner of Healy and Jones (1973) or along the lines of signal detection theory. When a priori probability varied, subjects in all instruction groups held constant the criteria defined by signal detection theory at the expense of shifting the criteria proposed by Healy and Jones. The results were taken as evidence for the psychological validity of recognition memory analyses based on signal detection theory.
\end{abstract}

Memory tasks are among the many situations that may lead the subject to adopt decision criteria. In a recall task in which the subject is not required to make a response for every item, he may formulate a criterion for deciding whether or not to respond. Similarly, in a recognition test in which the subject rates how confident he is that each item is old, he may set criteria for deciding which rating to give to each item. (For a task with $\mathrm{n}$ ratings, the subject would formulate $\mathrm{n}-1$ criteria, presumably all of the same general type but differing in strictness.) The present paper is concerned with determining the sort of criteria the subject adopts for such decisions. This paper also examines a more detailed question. Whatever criterion the subject chooses, can he hold it constant across changes in an independent task variable? For example, if the proportion of old items among all items tested is varied in a recognition task, will the subject be able to maintain a constant criterion despite changes in this proportion?

Several investigators have applied signal detection theory to such problems in memory research (e.g., Murdock, 1966). Signal detection analysis assumes that the state of an item can be represented by a value on the likelihood ratio scale, which scale for simplicity may be thought of as a familiarity continuum in a recognition task. Old and new items are assumed to form two overlapping normal distributions along this scale. The subject's criterion is taken to be indexed by the parameter beta, the cutoff point on the likelihood ratio scale. Beta reflects the ratio of the subject's hit rate (HR) to his false alarm rate (FAR), a ratio of conditional probabilities. ${ }^{1}$ The subject's criterion is called "strict" or

This research was supported by PHS Grants GM 1789 and GM 16735 to The Rockef eller University. The authors gratefully acknowledge the help and encouragement of Professor $W$. $K$. Estes. The authors also thank $M_{0}$ Kubovy and $J$. B. Healy for helpful suggestions. The order of authorship was determined alphabetically. "lax" depending on whether beta, or the corresponding HR/FAR, has a high or low value.

Healy and Jones (1973) have argued for a different view of the criterion. They propose that the most plausible psychological interpretation of instructions to maintain a given criterion involves the ratio of the proportion of hits $[\mathrm{P}(\mathrm{H})]$ to the proportion of false alarms $[\mathrm{P}(\mathrm{FA})]$, a ratio of joint probabilities. Specifically, it is argued that when instructed to maintain a strict criterion, the subject allows himself very few false alarms relative to the number of hits he makes, whereas, when instructed to operate with a lax criterion, the subject allows many false alarms relative to the number of his hits.

Healy and Jones note that for a constant a priori probability of an old item in a recognition task (or probability of a signal in a signal detection task or probability of a correct response in a Type $\mathrm{II}^{2}$ recall task) beta does indicate how many false alarms the subject allows himself to make relative to the number of his hits. When a priori probability is constant, the ratio of conditional probabilities, HR/FAR, is directly proportional to the ratio of joint probabilities, $\mathrm{P}(\mathrm{H}) / \mathrm{P}(\mathrm{FA})$. In most signal detection studies (e.g., Swets, Tanner, \& Birdsall, 1961), the probability of signal is not changed within a given session; however, it is common for the probability of a correct response to vary within a single session of a recall task as a function, say, of retention interval. When a priori probability is not constant, HR/FAR is not directly proportional to $\mathrm{P}(\mathrm{H}) / \mathrm{P}(\mathrm{FA})$, so that the definition of the criterion as beta is no longer consistent with the idea that the criterion is sensitive to the ratio of hits to false alarms. Indeed, when a priori probability changes, beta can remain constant while the subject's decisions become either more strict or more lax in terms of the number of 
false alarms he allows for each hit that he makes. Conversely, beta can change with a change in a priori probability even though the subject is being neither more strict nor more lax as measured by the ratio of hits to false alarms.

In view of this inconsistency, Healy and Jones proposed a new index of the criterion which, unlike beta, does reflect $\mathrm{P}(\mathrm{H}) / \mathrm{P}(\mathrm{FA})$. In a recognition task with $n$ confidence ratings $\left[R_{n}, R_{n-1}, \ldots, R_{1}(n \geqslant 2)\right]$, this index for the highest $\left(n^{\text {th }}\right)$ criterion is $P\left(\right.$ old $\left.\mid R_{n}\right)$, the a posteriori probability of an item being old, given that the highest rating $R_{n}$ was used. (In signal detection the index is $P\left(\right.$ signal $\left.\mid R_{n}\right)$; in Type II recall it is $P\left(\right.$ correct $\left.\mid R_{n}\right)$.] More generally, the index for the $j^{\text {th }}$ criterion $(2 \leqslant j \leqslant n)$ is $P\left(\right.$ old $\left.\mid R_{n} \vee R_{n-1} \vee \ldots v R_{j}\right)$. This index, which will be referred to here as the "strictness" index, changes or remains constant just when $\mathrm{P}(\mathrm{H}) / \mathrm{P}(\mathrm{FA})$ does; furthermore, this index has intuitive appeal. For example, it seems reasonable to demand that a subject hold constant the probability that an item he gives the highest rating is in fact an old item (or a signal or a correct response). That is, when the a priori probability of an old item changes, a subject may change the number of items he rates $R_{n}$, but those items rated $R_{n}$ should be just as likely to be old as before the change in a priori probability.

When a priori probability varies, the subject cannot maintain both criterion indices constant. Either beta or the strictness index must shift, as seen from the following equations for the case of the highest criterion in a recognition task:

$$
\frac{P(H)}{P(F A)}=\frac{P\left(R_{n} \& \text { old }\right)}{P\left(R_{n} \& \text { new }\right)}=\frac{P\left(R_{n} \mid \text { old }\right)}{P\left(R_{n} \mid \text { new }\right)} \cdot \frac{P(\text { old })}{P(\text { new })}=\frac{H R}{F A R} \cdot \frac{P(\text { old })}{P(\text { new })} .
$$

Clearly, if the ratio of a priori probabilities, $\mathrm{P}$ (old)/P(new), changes and the subject is attempting to keep $\mathrm{P}(\mathrm{H}) / \mathrm{P}(\mathrm{FA})$ (and, hence, the strictness index) constant, he must change HR/FAR (and, hence, beta), or if he is trying to hold HR/FAR constant, he must change $\mathrm{P}(\mathrm{H}) / \mathrm{P}(\mathrm{FA})$.

The analysis by Healy and Jones raises the possibility that the subject interprets standard instructions about confidence ratings to mean that he should adopt criteria corresponding to particular levels of the strictness index, rather than of beta. How subjects interpret instructions about confidence ratings has not been studied directly; consequently, the present study systematically investigates this question. In addition, this study continues the examination by Healy and Jones of the effects of changes in a priori probability on criterion levels, with specific reference to whether subjects naturally maintain either beta or the strictness index constant. The present research also examines whether explicit instructions and relevant feedback can induce a subject to hold either criterion index constant despite changes in a priori probability.

\section{EXPERIMENT I}

Since the a priori probability of a correct response in a recall task is not under direct experimenter control, both experiments reported here use a recognition memory paradigm, permitting direct manipulation of the a priori probability of an old item. Experiment I examines the effect of type of criterion instruction and feedback when a priori probability is held constant for each subject.

\section{Method}

Subjects. The subjects were 24 young adults solicited through newspaper advertisements and paid for their participation at the rate of $\$ 2.50 / \mathrm{h}$. Each subject was run individually for one session approximately $1 \mathrm{~h}$ long. Depending on his time of arrival for testing, each subject was assigned to one of three instruction groups, with eight subjects in each group.

Apparatus. A VT05 display screen with attached typewriter keyboard, controlled by a PDP-8/I computer, was used for the visual presentation of each block of trials as well as for the subject's manual responses. All displays were centered on the screen. The required responses, "1," " 2 ," " 3 ," and "4," were located in the standard typewriter keyboard position.

Design and Procedure. Each subject's session included eight blocks of trials, with a rest period after the first four. Each block consisted of a study and a test phase. During the study phase, the subject saw 20 three-digit numbers to be remembered; then during the test phase, he rated each of 40 three-digit numbers as to how certain he was that it had been shown previously.

The 40 test items in each block included the currect 20 study items (old items) along with 20 distractors (new items). Since new sets of both old and new items were used for each block, each old item appeared exactly twice (once at study and once at test), and each new item was shown exactly once during the eight-block session. The set of items used was chosen at random without replacement from the numbers 100-999, and each subject was shown this set of items in one of eight random orders. A different order was used for each of the eight subjects in an instruction group.

Two types of feedback were employed. At the end of every block, subjects in two of the three instruction groups were given block feedback concerning the criterion levels they maintained for that block (see below). Furthermore, all subjects were given item feedback; immediately after each rating response the subject saw whether the given test item had been in fact old or new. In addition to feedback, all subjects received information at the start of each test phase as to the numbers of old and new test items that would be shown. (In this experiment, as noted above, these values were held constant at 20 old and 20 new items.)

The timing within each trial block was as follows: To start, the word "study" was shown on the screen for $3 \mathrm{sec}$, followed by a $1 / 2-\sec$ blank. Next appeared the 20 study items, each displayed for $2 \mathrm{sec}$ and followed by a $1 / 2$-sec blank. After the 20 th study item and blank, the word "test" was shown for $3 \mathrm{sec}$ and followed by another $3 / 2$-sec blank. Then the following message was displayed for $6 \mathrm{sec}$ : "You will see 40 test items. 20 will be old items. 20 will be new items." After a 1-sec blank, the 40-item test sequence began. Each test item remained on the screen until the subject made an appropriate response. If the subject hit an irrelevant key, the message "You did not respond with $1,2,3$, or 4 . Try again." was shown below the test stimulus for $2 \mathrm{sec}$. As soon as the subject made an appropriate response, the test number was replaced by the feedback word "old" or "new" for $1 \mathrm{sec}$, followed immediately by a new test stimulus. 
After the 40 th test item and its feedback, the subject was given appropriate block feedback or saw a blank screen (see below) for about $10 \mathrm{sec}$. Following a $1 / 2-\mathrm{sec}$ blank, the next block began with the word "study."

The procedures for the three different instruction groups varied only in the instructions regarding criteria and in the biock feedback displayed. Subjects in the "standard" group were given standard instructions about the ratings. In contrast, subjects in the "strictness index" group were explicitly asked to hold the strictness index at three specified levels, and subjects in the "HR/FAR" group were explicitly asked to hold HR/FAR at three specified levels. In particular, all instructions included the following request: "For each test item that appears on the screen you will indicate whether you think the item is old or new. You will do this by typing ' 1 ,' ' 2 ,' ' 3 ,' or ' 4 ,' depending on how certain you are that the test item is old or new-with ' 4 ' meaning most certain it is old and ' 1 ' meaning most certain it is new. More specifically,...." Here the instructions diverged. In the standard condition, subjects were told: "If you are certain that the item is old, you should type '4.' If you think the item is old but are not entirely certain, you should type '3.' On the other hand, if you are certain that the item is new, you should type ' 1 .' If you think the item is new but are not entirely certain, you should type '2'." In the strictness index condition, subjects were told: "You are to make sure that $90 \%$ of the items that you rate ' 4 ' are actually old items, $75 \%$ of the items that you rate ' 3 ' or higher are actually old items, and $60 \%$ of the items that you rate ' 2 ' or higher are actually old items." Subjects in the HR/FAR condition were told: "You are to make sure that the probability that you rate an old item as ' 4 ' is 9 times as high as the probability that you rate a new item as '4.' Also, make sure that the probability that you rate an old item as ' 3 ' or higher is 3 times as high as the probability that you rate a new item as ' 3 ' or higher. And, finally, make sure that the probability that you rate an old item as '2' or higher is 1.5 times as high as the probability that you rate a new item as ' 2 ' or higher."

Note that the designated levels of the two criterion indices are equivalent when the a priori probability of an old item is $1 / 2$, as was the case in each block of the present experiment. Hence, the strictness index and the HR/FAR groups differed not in the criterion levels prescribed but only in the instructions describing those levels.

The block feedback differed for the three instruction groups. The subjects in the standard group were given no block feedback; they saw a blank screen during the 10-sec block feedback period. In contrast, the subjects in the strictness index group saw the following message: "Probability that item was old when you rated it: $4 ; 3$ or $4 ; 2,3$, or 4 . Ideal value: $90.0 \%$, $75.0 \%, 60.0 \%$. Your actual value: XX.X\%, XX.X\%, XX.X\%," with the ideal and actual values for a given rating shown directly below that rating. Subjects in the HR/FAR group saw the following message: "Probability that you gave high ratings to old items, divided by probability that you gave high ratings to new items, for these high ratings: $4 ; 3$ or $4 ; 2,3$, or 4 . Ideal value: $9.00,3.00,1.50$. Your actual value: X.XX, X.XX, X.XX," with the ideal and actual values for a given rating shown directly below that rating.

\section{Results}

Of initial interest is whether subjects in fact differentiated the four rating levels as instructed. Accordingly, the probability of an item rated $R_{\mathbf{j}}$ being an old item was computed for each subject, for each of the four rating levels, averaging across blocks. (Note that these a posteriori probabilities do not correspond to the strictness criterion indices except for the highest
Table 1

Probability of Old Item as a Function of Rating for Experiment I

\begin{tabular}{lcccc}
\hline & \multicolumn{4}{c}{ Mean P(Old IRating) } \\
\cline { 2 - 5 } $\begin{array}{l}\text { Instruction } \\
\text { Group }\end{array}$ & 1 & 2 & 3 & 4 \\
\hline Standard & .34 & .40 & .43 & .65 \\
Strictness & .32 & .46 & .58 & .68 \\
HR/FAR & .28 & .42 & .51 & .74 \\
\hline
\end{tabular}

criterion.) The mean values are shown in Table 1. Differences among the four rating levels are evident for all three instruction groups. A multifactorial analysis of variance with repeated measures performed on these data found the factor of rating level to be significant $[F(3,63)=63.0, p<.01]$, as well as the factor of groups $[F(2,21)=5.7, p<.05]$, but not the interaction of the two factors $[F(6,63)=1.7, p>.10]$.

Both criterion indices, the strictness index and the HR/FAR index, were computed for all eight blocks of every subject. (For any block in which a subject had a FAR of zero and a HR greater than zero, the HR/FAR was taken to be 20.0.) Table 2 shows the mean values of the criterion indices for the three criterion levels (the strict criterion separating ratings 3 and 4 , the medium criterion separating ratings 2 and 3 , and the lax criterion separating ratings 1 and 2) as a function of block serial position and instruction group. Six multifactorial analyses of variance with repeated measures were performed on the data, one analysis for each criterion level of each criterion index (strict, medium, and lax levels of the strictness index and strict, medium, and lax levels of the HR/FAR index).

Two aspects of the data are noteworthy. First, there is little consistent change in criterion levels across blocks, except for a slight decrease at the last block. This result is remarkable in light of the fact that no practice trials were included. The analyses of variance, however, do find the factor of blocks significant or barely significant for the lower criterion levels. (For the strictness index, on the strict criterion, $\mathrm{F}=2.0, \mathrm{p}>.05$; on the medium criterion, $\mathrm{F}=2.1, \mathrm{p} \approx .05$; on the lax criterion, $\mathrm{F}=3.5$, $\mathrm{p}<.01$; for the HR/FAR index, on the strict criterion, $\mathrm{F}=1.6, \mathrm{p}>.10 ;$ on the medium criterion, $\mathrm{F}=2.4$, $\mathrm{p}<.05 ;$ on the lax criterion, $\mathrm{F}=3.2, \mathrm{p}<.01$; $\mathrm{df}=7,147$ in each case.) At least for the strict criterion, these data do not justify rejecting the hypothesis that subjects are able to maintain a constant criterion across blocks when a priori probability is held constant.

The second noteworthy aspect of the data is the similarity of the three instruction groups in terms of the values of the criterion indices. Not one of the six analyses of variance found the factor of instruction group slgnificant $(p>.10$ in each case). The finding of no difference between the standard and the strictness index groups is consistent with the proposal by Healy 
Table 2

Mean Criterion Index Values for Experiment I by Blocks

\begin{tabular}{|c|c|c|c|c|c|c|c|c|c|c|}
\hline \multirow{2}{*}{$\begin{array}{c}\text { Criterion } \\
\text { Level }\end{array}$} & \multirow{2}{*}{$\begin{array}{c}\text { Instruction } \\
\text { Group }\end{array}$} & \multicolumn{9}{|c|}{ Block Position } \\
\hline & & 1 & 2 & 3 & 4 & 5 & 6 & 7 & 8 & All \\
\hline \multicolumn{11}{|l|}{ Strictness Index } \\
\hline Strict & $\begin{array}{l}\text { Standard } \\
\text { Strictness } \\
\text { HR/FAR }\end{array}$ & $\begin{array}{l}70.2 \\
67.2 \\
72.2\end{array}$ & $\begin{array}{l}67.0 \\
61.4 \\
67.5\end{array}$ & $\begin{array}{l}67.6 \\
66.8 \\
76.7\end{array}$ & $\begin{array}{l}71.4 \\
71.1 \\
77.0\end{array}$ & $\begin{array}{l}61.3 \\
70.5 \\
79.2\end{array}$ & $\begin{array}{l}64.1 \\
67.0 \\
83.5\end{array}$ & $\begin{array}{l}73.0 \\
72.7 \\
80.7\end{array}$ & $\begin{array}{l}59.2 \\
67.4 \\
64.6\end{array}$ & $\begin{array}{l}66.7 \\
68.0 \\
75.2\end{array}$ \\
\hline Medium & $\begin{array}{l}\text { Standard } \\
\text { Strictness } \\
\text { HR/FAR }\end{array}$ & $\begin{array}{l}60.2 \\
66.1 \\
65.3\end{array}$ & $\begin{array}{l}64.6 \\
61.6 \\
61.5\end{array}$ & $\begin{array}{l}60.3 \\
64.3 \\
64.5\end{array}$ & $\begin{array}{l}68.7 \\
66.9 \\
73.1\end{array}$ & $\begin{array}{l}59.1 \\
66.6 \\
63.0\end{array}$ & $\begin{array}{l}57.5 \\
63.7 \\
71.1\end{array}$ & $\begin{array}{l}60.6 \\
67.5 \\
66.5\end{array}$ & $\begin{array}{l}54.4 \\
62.2 \\
64.2\end{array}$ & $\begin{array}{l}60.7 \\
64.9 \\
66.1\end{array}$ \\
\hline Lax & $\begin{array}{l}\text { Standard } \\
\text { Strictness } \\
\text { HR/FAR }\end{array}$ & $\begin{array}{l}55.5 \\
57.3 \\
55.3\end{array}$ & $\begin{array}{l}55.7 \\
57.5 \\
55.5\end{array}$ & $\begin{array}{l}56.5 \\
58.8 \\
55.9\end{array}$ & $\begin{array}{l}61.7 \\
65.9 \\
61.5\end{array}$ & $\begin{array}{l}51.7 \\
60.1 \\
59.1\end{array}$ & $\begin{array}{l}56.8 \\
57.3 \\
59.5\end{array}$ & $\begin{array}{l}55.7 \\
60.4 \\
59.9\end{array}$ & $\begin{array}{l}52.9 \\
59.6 \\
59.4\end{array}$ & $\begin{array}{l}55.8 \\
59.6 \\
58.3\end{array}$ \\
\hline \multicolumn{11}{|l|}{ HR/FAR Index } \\
\hline Strict & $\begin{array}{l}\text { Standard } \\
\text { Strictness } \\
\text { HR/FAR }\end{array}$ & $\begin{array}{l}4.70 \\
2.46 \\
3.94\end{array}$ & $\begin{array}{l}2.86 \\
2.73 \\
2.94\end{array}$ & $\begin{array}{l}2.59 \\
2.33 \\
8.95\end{array}$ & $\begin{array}{l}3.65 \\
4.48 \\
8.00\end{array}$ & $\begin{array}{l}2.04 \\
2.99 \\
5.46\end{array}$ & $\begin{array}{l}2.00 \\
4.03 \\
7.23\end{array}$ & $\begin{array}{l}4.65 \\
4.82 \\
8.08\end{array}$ & $\begin{array}{l}1.83 \\
2.78 \\
5.28\end{array}$ & $\begin{array}{l}3.04 \\
3.33 \\
6.24\end{array}$ \\
\hline Medium & $\begin{array}{l}\text { Standard } \\
\text { Strictness } \\
\text { HR/FAR }\end{array}$ & $\begin{array}{l}1.56 \\
2.29 \\
2.43\end{array}$ & $\begin{array}{l}2.11 \\
1.72 \\
1.85\end{array}$ & $\begin{array}{l}1.67 \\
1.88 \\
2.81\end{array}$ & $\begin{array}{l}3.51 \\
2.34 \\
4.86\end{array}$ & $\begin{array}{l}1.56 \\
2.14 \\
1.77\end{array}$ & $\begin{array}{l}1.46 \\
3.23 \\
3.86\end{array}$ & $\begin{array}{l}1.58 \\
2.78 \\
2.49\end{array}$ & $\begin{array}{l}1.25 \\
1.69 \\
2.09\end{array}$ & $\begin{array}{l}1.84 \\
2.26 \\
2.77\end{array}$ \\
\hline Lax & $\begin{array}{l}\text { Standard } \\
\text { Strictness } \\
\text { HR/FAR }\end{array}$ & $\begin{array}{l}1.27 \\
1.47 \\
1.26\end{array}$ & $\begin{array}{l}1.31 \\
1.43 \\
1.32\end{array}$ & $\begin{array}{l}1.40 \\
1.54 \\
1.34 \\
\end{array}$ & $\begin{array}{l}1.76 \\
2.05 \\
1.69\end{array}$ & $\begin{array}{l}1.10 \\
1.65 \\
1.48\end{array}$ & $\begin{array}{l}1.34 \\
1.38 \\
1.66\end{array}$ & $\begin{array}{l}1.28 \\
1.62 \\
1.54\end{array}$ & $\begin{array}{l}1.15 \\
1.57 \\
1.62\end{array}$ & $\begin{array}{l}1.32 \\
1.59 \\
1.49\end{array}$ \\
\hline
\end{tabular}

and Jones (1973) that subjects interpret standard instructions in terms of the strictness index. However, the parallel finding of no difference between the standard and the HR/FAR groups does not rule out the possibility that subjects interpret the standard instructions in terms of beta. These findings preclude a conclusion about subjects' interpretations of standard instructions.

\section{EXPERIMENT II}

Experiment II was performed to study further how subjects interpret the standard instructions; in addition, the a priori probabilities were varied within each subject's session in this experiment in order to determine whether the subject can hold constant criteria across blocks when a priori probability is changed.

\section{Method}

Subjects. Twenty-four new subjects were recruited and divided into three instruction groups of eight subjects each, just as in Experiment $I$.

Apparatus, Design, and Procedure. The apparatus, design, procedure, and instructions of Experiment II were identical to those of Experiment I with the following exception: In the present experiment, the a priori probability of an old item was $1 / 2$ for four of each subject's eight blocks and $1 / 4$ for the other four. Specifically, four of the blocks gave 40-item test sequences consisting of 20 old and 20 new items, whereas the other four blocks gave 40-item test sequences consisting of 10 old and 30 new items. These two a priori probabilities were assigned to each subject's blocks in one of eight pseudorandom orders, constrained so that no three successive blocks had the same a priori probability of an old item. Furthermore, the set of eight pseudorandom orders included four orders and their respective inverses; for example, $1 / 2,1 / 4,1 / 4,1 / 2,1 / 2,1 / 4,1 / 2,1 / 4$ was the order of a priori probabilities across blocks for one subject, and its inverse, $1 / 4,1 / 2,1 / 2,1 / 4,1 / 4,1 / 2,1 / 4,1 / 2$, was the block order for another subject. Each of the eight block orders was used for three subjects, one in each instruction group.

Subjects were told at the start of each test sequence how many old and how many new items would be shown; clearly, this information was not the same for all blocks in the present experiment as it was in Experiment I. Finally, note that, since every block included 20 study items but not necessarily 20 old test items, some old items appeared only once in the present experiment, unlike Experiment I.

\section{Results}

Of initial interest again is whether subjects were able in this experiment, as in the previous one, to differentiate the four rating levels. The probability of an old item was computed for each subject, for each of the four ratings, averaging across blocks with the same a priori probability. The mean values are shown in Table 3, and differences among the four rating levels are

Table 3

Probability of Old Item as a Function of Rating for Experiment II

\begin{tabular}{|c|c|c|c|c|c|}
\hline \multirow{3}{*}{$\begin{array}{l}\text { Instruction } \\
\text { Group }\end{array}$} & \multirow{3}{*}{$\begin{array}{l}\quad \text { Mean } \\
\text { A Priori } \\
\text { Probabil: } \\
\text { ity Level }\end{array}$} & \multirow{2}{*}{\multicolumn{4}{|c|}{ Rating }} \\
\hline & & & & & \\
\hline & & 1 & 2 & 3 & 4 \\
\hline \multirow{2}{*}{ Standard } & $1 / 2$ & .35 & .38 & .43 & .73 \\
\hline & $1 / 4$ & .17 & .16 & .47 & .46 \\
\hline \multirow{2}{*}{ Strictness } & $1 / 2$ & .29 & .43 & .58 & .78 \\
\hline & $1 / 4$ & .15 & .21 & .32 & .63 \\
\hline \multirow{2}{*}{ HR/FAR } & $1 / 2$ & .33 & .46 & .50 & .75 \\
\hline & $1 / 4$ & .13 & .21 & .33 & .53 \\
\hline
\end{tabular}


again evident for all three instruction groups at both levels of a priori probability. An analysis of variance performed on the data showed the factor of rating level to be significant $[F(3,63)=55.2, p<.01]$, as well as the factor of a priori probability $[\mathrm{F}(1,21)=148.5, \mathrm{p}<.01]$, but not the factor of groups $[F(2,21)=.6, p>.10]$. The only interaction among these factors that reached significance was the three-way interaction $[F(6,63)=$ $2.6, \mathrm{p}<.05]$.

Both criterion indices (strictness and HR/FAR) were computed for all subjects. Table 4 shows the mean values of the criterion indices for the three criterion levels as a function of a priori probability and instruction group. Six analyses of variance were performed on the data, as on the analogous data of Experiment I.

It is clear from Table 4 that in Experiment II, as in Experiment I, there is little difference between the three instruction groups. The factor of instruction group was not significant in any of the six analyses $(p>.10$ in each case). The data again preclude a conclusion about how subjects interpreted the standard instructions.

The data are conclusive on the question of which criterion index subjects held constant across different a priori probability levels. Clearly, subjects were unable to hold constant the strictness index when a priori probability changed. This result holds even for the subjects in the strictness index group, who were explicitly instructed to hold the strictness index at fixed levels. According to the analyses of variance, the factor of a priori probability was highly significant for each of the three criterion levels when the strictness index was considered [for the strict criterion, $F(1,21)=83.1$; for the medium criterion, $F(1,21)=125.3$; for the lax criterion, $\mathrm{F}(1,21)=290.6 ; \mathrm{p}<<.01$ in each case $]$. In contrast, all subjects, including those in the strictness index group, did hold HR/FAR relatively constant. In only one analysis of variance was the factor of a priori probability significant for the HR/FAR index, and in that case it was only barely significant [for the strict criterion, $F(1,21)=.3, \quad \mathrm{p}>.10 ;$ for the medium criterion, $F(1,21)=3.7, p>.05$; for the lax criterion, $\mathrm{F}(1,21)=4.5, \mathrm{p}<.051$.

As a control measure to verify that the observed differential effects of a priori probability were not due to any variable associated with block position, each subject in Experiment I was randomly paired with a subject in Experiment II. The criterion index values were computed for subjects in Experiment I by averaging across blocks in which the matched subjects in Experiment II had a priori probability equal to either $1 / 2$ or $1 / 4$. A series of six analyses of variance was performed on the data, one analysis for each criterion level of each criterion index, and no analysis found the factor of a priori probability significant $(F<1.00$ in each case).
Table 4

Mean Criterion Index Values for Experiment II

\begin{tabular}{clll}
\hline & & \multicolumn{2}{c}{$\begin{array}{c}\text { A Priori Probability } \\
\text { of Old Item }\end{array}$} \\
\cline { 4 - 4 } $\begin{array}{c}\text { Criterion } \\
\text { Level }\end{array}$ & $\begin{array}{c}\text { Instruction } \\
\text { Group }\end{array}$ & $1 / 2$ & $1 / 4$ \\
\hline Strictness Index & & & \\
Strict & Standard & 73.3 & 47.6 \\
& Strictness & 81.9 & 62.5 \\
& HR/FAR & 76.6 & 55.3 \\
Medium & Standard & 67.2 & 43.2 \\
& Strictness & 69.7 & 49.5 \\
& HR/FAR & 66.1 & 43.8 \\
Lax & Standard & 62.3 & 39.6 \\
& Strictness & 58.2 & 35.7 \\
HR/FAR Index & HR/FAR & 59.7 & 35.9 \\
& & & \\
Strict & Standard & 5.36 & 4.13 \\
& Strictness & 9.43 & 9.41 \\
& HR/FAR & 7.09 & 7.09 \\
Medium & Standard & 2.40 & 3.15 \\
& Strictness & 3.54 & 4.64 \\
& HR/FAR & 2.50 & 3.06 \\
& Standard & 1.87 & 2.49 \\
Lax & Strictness & 1.46 & 1.84 \\
& HR/FAR & 1.73 & 2.04 \\
\hline
\end{tabular}

The observation that subjects held HR/FAR relatively constant across a priori probability levels in Experiment II raises the question whether subjects held simply the ratio constant or both HR and FAR constant, as would be expected if they were maintaining a constant cutoff point (beta) on the likelihood ratio scale, as defined by signal detection theory. HR and FAR were separately computed for each subject, for each criterion level, yielding the mean values shown in Table 5. A series of six multifactorial analyses of variance with repeated measures was computed for the data, one analysis for each criterion level of HR and one for each level of FAR. For each criterion level, there was no significant difference in either HR or FAR as a function of a priori probability ( $p>.05$ in each case).

Table 5

Mean HR and FAR Values for Experiment II

\begin{tabular}{|c|c|c|c|c|c|}
\hline \multirow{2}{*}{$\begin{array}{c}\text { Criterion } \\
\text { Level }\end{array}$} & \multirow{2}{*}{$\begin{array}{l}\text { Instruction } \\
\text { Group }\end{array}$} & \multicolumn{2}{|c|}{$\begin{array}{c}\text { HR } \\
\text { A Priori } \\
\text { Probability }\end{array}$} & \multicolumn{2}{|c|}{$\begin{array}{c}\text { FAR } \\
\text { A Priori } \\
\text { Probability }\end{array}$} \\
\hline & & $1 / 2$ & $1 / 4$ & $1 / 2$ & $1 / 4$ \\
\hline \multirow{3}{*}{ Strict } & Standard & .52 & .49 & .23 & .23 \\
\hline & Strictness & .30 & .30 & .10 & .09 \\
\hline & HR/FAR & .39 & .32 & .14 & .12 \\
\hline \multirow{3}{*}{ Medium } & Standard & .62 & .59 & .32 & .31 \\
\hline & Strictness & .52 & .53 & .26 & .25 \\
\hline & HR/FAR & .59 & .54 & .31 & .25 \\
\hline \multirow{3}{*}{ Lax } & Standard & .71 & .66 & .46 & .41 \\
\hline & Strictness & .78 & .78 & .60 & .55 \\
\hline & HR/FAR & .74 & .71 & .53 & .48 \\
\hline
\end{tabular}


These findings are consistent with the notion that subjects are maintaining a constant cutoff point on the likelihood ratio scale.

\section{DISCUSSION AND CONCLUSIONS}

It is evident that in a task of this sort, if criteria are defined in terms of HR/FAR, subjects not only are able to hold their criteria relatively constant but do so even when given explicit instructions to the contrary, as were subjects in the strictness index group. Additional analyses revealed that subjects not only maintain the ratio HR/FAR constant across changes in a priori probability but also separately maintain both the HR and the FAR constant. This finding is consistent with the idea that subjects maintain a constant cutoff point (beta) on the likelihood ratio scale, as defined by signal detection theory. The results give support for the psychological validity of analyses of recognition memory tasks along the lines of signal detection theory. In contrast, it is clear that subjects are unable to maintain constant criteria defined in terms of the strictness index, even when given criterion feedback and explicit knowledge of a priori probabilities.

The failure to find a significant effect of instruction group in either experiment suggests that subjects interpret all sets of instructions in the same way as standard instructions and are unable in the duration of a short session to make effective use of block feedback. Therefore, no conclusion can be reached as to how subjects interpret standard instructions. Subjects may or may not interpret the instructions in terms of the strictness index as Healy and Jones (1973) have suggested. Although the negative results of the present study cannot settle this question, they do have an important methodological implication; namely, it is difficult to instruct naive subjects which criteria to adopt in a task of this sort.

Early work in signal detection theory (e.g., Swets, Tanner, \& Birdsall, 1961) yielded a formula for the value of beta that a subject should adopt in order to optimize his performance in terms of costs and benefits. By the formula, the optimal value of beta changes as the a priori probability changes, assuming constant costs and benefits. Thus, subjects in the present situation are not behaving optimally when they maintain a constant beta across changes in a priori probability levels (except, of course, in the HR/FAR instruction group, where they are explicitly told to hold HR/FAR and, hence, beta constant). The performance of subjects in the present situation is then markedly different from that in the signal detection study by Swets et al. (1961). In that study, as a priori probability changed, subjects changed their HRs and FARs in the direction specified by the formula for the optimal beta. The primary difference between the two studies lies in the paradigms employed: Experiment II involved a recognition memory task, whereas the earlier study involved a signal detection task. Lieblich and Lieblich (1969) have argued that the task is an important variable in determining the extent of shift in decision patterns. In their study, Lieblich and Lieblich were unable to induce subjects to shift decision patterns with changes in payoffs, and the Lieblichs attribute their difficulty to the nature of their decision task, which involved arithmetical estimation. However, two other differences may be more critical in explaining why shifts in beta occurred in the signal detection study but not in the present study. These differences are the rate of change in conditions and the extent of subjects' training. Conditions (a priori probability levels and/or payoffs) changed between sessions in the earlier study but within sessions in the present study (and in the study by Lieblich and Lieblich). Furthermore, subjects were highly practiced in the earlier study but not in the present study (nor in that of the Lieblichs). These facts suggest that relatively untrained subjects are unable to shift beta within the duration of a single short session.

\section{REFERENCES}

Clarke, F. R., Birdsall. T. G., \& Tanner, W. P., Jr. Two types of ROC curves and definitions of parameters. Journal of the Acoustical Society of America, 1959, 31, 629-630.

Healy, A. F., \& Jones, C. Criterion shifts in recall. Psychological Bulletin, $1973,79,335-340$.

Lieblich, I., \& Lieblich, A. Effects of different payoff matrices on arithmetical estimation tasks: An attempt to produce "rationality." Perceptual and Motor Skills, 1969, 29, 467-473. Murdock, B. B., Jr. The criterion problem in short-term memory. Journal of Experimental Psychology, 1966, 72, 317-324.

Swets, J. A., Tanner, W. P., Jr., \& Birdsall, T. G. Decision processes in perception. Psychological Review, 1961,68, 301-340.

\section{NOTES}

1. It can be shown that for fixed distributions, as beta increases so does the ratio HR/FAR. HR and FAR are the areas to the right of beta under the normal distributions representing old and new items, respectively. When the distribution of old items has variance no less than that of the distribution of new items, the proof follows directly by showing that the derivative of HR/FAR with respect to beta is positive.

2. See Clarke, Birdsall, and Tanner (1959) and Healy and Jones (1973).

(Received for publication May 11, 1974; revision received August 13, 1974.) 